\title{
Analysis of femtosecond stimulated Raman spectroscopy of excited-state evolution in bacteriorhodopsin
}

\author{
Kai Niu, ${ }^{1}$ Bin Zhao, ${ }^{1}$ Zhigang Sun, ${ }^{2}$ and Soo-Y. Lee ${ }^{1, a)}$ \\ ${ }^{1}$ Division of Physics and Applied Physics and Division of Chemistry and Biological Chemistry, \\ School of Physical and Mathematical Sciences, Nanyang Technological University, Singapore 637371, \\ Singapore \\ ${ }^{2}$ Department of Chemistry, Duke University, Durham, North Carolina 27708-0354, USA
}

(Received 27 November 2009; accepted 2 February 2010; published online 25 February 2010)

\begin{abstract}
The dispersive lines observed in time-resolved femtosecond stimulated Raman spectroscopy (FSRS), using a pair of $809 \mathrm{~nm}, 3$ ps Raman pump, and 840-960 nm ultrashort probe pulse, for the first 500 fs photoisomerization dynamics in the excited state of bacteriorhodopsin, BR* $\left(S_{1}\right)$, created by a prior $500 \mathrm{~nm}, 35 \mathrm{fs}$ actinic pump pulse, have previously been attributed to Raman initiated by nonlinear emission (RINE). We used four-wave mixing energy level diagrams to describe the FSRS process, which include RINE as a subset, and a 29-mode harmonic oscillator model for $\mathrm{BR}_{568}$ in the calculations. Our calculations showed that FSRS of $\mathrm{BR}^{*}$ effectively occurs from the ground vibrational state of each of the observed $800-1800 \mathrm{~cm}^{-1}$ modes of $S_{1}$. The lifetime on $S_{1}$ determines the linewidth and decay of the dispersive lines, and is estimated to be $\sim 600 \mathrm{fs}$, comparable to the stimulated emission decay time. The FSRS dipole couplings are from the ground vibrational state of $S_{1}$ to high energy vibrational states on $B R\left(S_{0}\right)$, and we place a fast decay lifetime of $\sim 100 \mathrm{fs}$ on $\mathrm{S}_{0}$ which can be attributed to the correlation function from the many unobserved low frequency modes. The FSRS dispersive lines are shown to be due to the inverse Raman scattering term with $|0\rangle\langle 1|$ vibrational coherence on the $S_{1}$ surface, and are not due to RINE with vibrational coherence on the $\mathrm{S}_{0}$ surface. Our calculations show that the RINE process gives rise to broad featureless spectra. (C) 2010 American Institute of Physics. [doi:10.1063/1.3330818]
\end{abstract}

\section{INTRODUCTION}

The photophysics and photochemistry of retinal proteins, which are important photosynthetic systems, have been studied extensively in a quest to understand the molecular mechanism by which visible light is initially stored with accompanying structural changes in the retinal chromophore and how this energy is used by the protein for a specific function while returning the retinal chromophore to its original state. ${ }^{1-5}$ Bacteriorhodopsin (BR), for example, is a protein found in the purple membrane of Halobacterium salinarum, which functions as a light activated proton pump. Upon absorption of a visible photon, the all-trans retinal chromophore in BR is instantaneously excited to $\mathrm{BR}^{*}$ in the Franck-Condon region, often labeled as $\mathrm{H}$, and in $\sim 200 \mathrm{fs}$ the excited state wave packet moves to an intermediate $\mathrm{I}_{460}$, then to a $\mathrm{J}_{625}$ intermediate in $\sim 500 \mathrm{fs}$, and subsequently to the fully isomerized 13-cis retinal $\mathrm{K}_{610}$ product in $\sim 3 \mathrm{ps}$ (Fig. 1). ${ }^{2,3}$ Further changes in the microsecond and millisecond time regime then lead to a transport of a proton out of the cell and the regeneration of all-trans retinal. ${ }^{6}$ The ultrafast primary events in the temporal and structural changes in BR upon light absorption have to be studied with femtosecond transient electronic and vibrational spectroscopies.

Femtosecond stimulated Raman spectroscopy (FSRS) with femtosecond time resolution and high vibrational spec-

\footnotetext{
${ }^{a)}$ Author to whom correspondence should be addressed. Electronic mail: sooying@ntu.edu.sg.
}

tral resolution has been developed to study time-resolved structural changes in ultrafast photophysical and photochemical processes. ${ }^{7-17}$ In the time-resolved experiments, three pulses are used: a $<50$ fs actinic pump pulse to excite the molecule to an upper electronic state, followed by stimulated Raman spectroscopy with an overlapping pair of ps Raman pump pulse, and a $\sim 15$ fs continuum probe pulse. It is possible to acquire spectra with time resolution of $\sim 50 \mathrm{fs}$ in the time delay between the actinic pump and probe pulses, and spectral resolution of $<10 \mathrm{~cm}^{-1}$ due to the picosecond Raman pump pulse. ${ }^{13,15}$ Other advantages of FSRS are rapid data acquisition, Raman spectra unaffected by background fluorescence, and vibrational spectrum acquisition in the range of $600-2500 \mathrm{~cm}^{-1}$. FSRS has been used to study numerous systems, including vibrational relaxation and internal conversion in $\beta$-carotene ${ }^{10}$ and diphenyloctatetraene, ${ }^{8}$ excited state structural dynamics of the photoisomerization of the retinal chromophore in $\mathrm{BR}^{2,11}$ and rhodopsin, ${ }^{12}$ direct observation of anharmonic coupling in $\mathrm{CDCl}_{3},{ }^{14}$ and excited state isomerization in phytochrome. ${ }^{18}$

Lee and co-workers ${ }^{19,20}$ developed the quantum theory for FSRS from both a stationary state and a moving wave packet prepared by an actinic pump pulse or by impulsive Raman scattering. It was shown that the FSRS spectrum in the probe pulse direction can be described by eight Feynman dual time-line diagrams and fall into four groups which are labeled as stimulated Raman scattering (SRS) or inverse Raman scattering (IRS)-SRS(I), SRS(II), IRS(I), and IRS(II). ${ }^{19}$ In particular, SRS(I) has three Feynman diagrams 


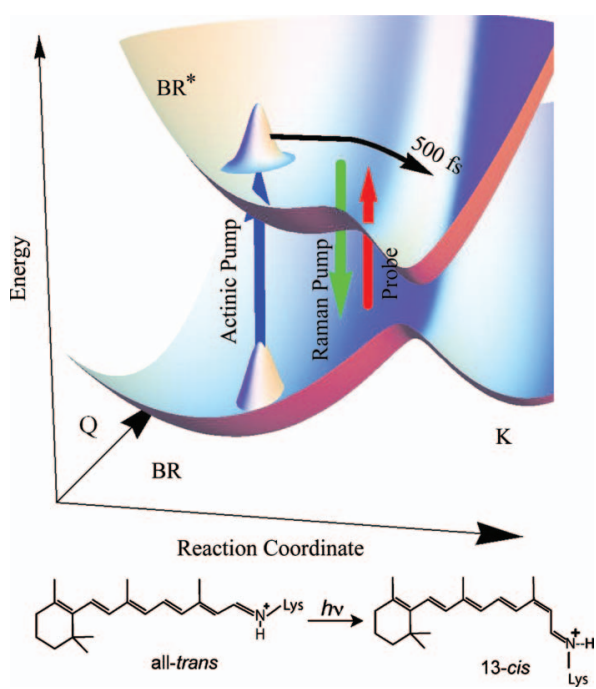

FIG. 1. Schematic diagram of the photoisomerization process of BR and the FSRS experiment. An ultrashort actinic pump pulse prepares a wave packet on $\mathrm{BR}^{*}$, and as the wave packet moves from the Franck-Condon region to other parts of the excited state surface and finally to the isomerized product it is investigated with a pair of picosecond Raman pump and femtosecond probe pulses.

corresponding to resonance Raman scattering (RRS) with no intermediate state population, and hot luminescence (HL) where intermediate population is possible in the excited state, similar to those described by $S^{2} n^{21}$ for spontaneous Raman scattering where the probe is now the vacuum field. For the other terms, SRS(II) also has three diagrams, while IRS(I) and IRS(II) have one each, where the role of the probe pulse is reversed from that for SRS(I). The quantum theory of FSRS has been used to successfully explain the inversion symmetry in the FSRS Stokes and anti-Stokes spectrum, ${ }^{19,20}$ to simulate off-resonance and resonance FSRS spectra of rhodamine $6 \mathrm{G}$ in comparison with experiment, ${ }^{22}$ and to simulate how FSRS can provide direct observation of anharmonic coupling in $\mathrm{CDCl}_{3}$. ${ }^{23}$

Recently, McCamant et al. ${ }^{11}$ used FSRS to study the photoisomerization dynamics in the excited state of BR. A mixture of ground state BR and excited state BR* was prepared by an actinic pump and both the FSRS of BR and BR* were recorded. The FSRS (Stokes) spectrum of ground state BR showed the expected Lorentzian lines at vibrational frequencies from $750-1700 \mathrm{~cm}^{-1}$ due to the SRS(I) process, but the FSRS spectra of the mixture of $B R$ and $\mathrm{BR}^{*}$ for actinic pump-probe delay times from 0-500 fs displayed dispersive looking lines at slightly downshifted $\left(\sim 1-5 \mathrm{~cm}^{-1}\right)$ vibrational frequencies. ${ }^{11}$ The dispersive lines in the FSRS spectra of $\mathrm{BR}^{*}$ were revealed by subtracting the baseline which could be caused by Raman pump induced bleach of the stimulated emission band and 0.91 times the FSRS spectrum of ground state BR. A "Raman initiated by nonlinear emission" (RINE) mechanism was postulated to account for the dispersive lines. ${ }^{11}$ In RINE, ground state vibrational coherence was first generated by a set of dipole couplings due to the Raman pump and probe fields acting on the ket and bra from the excited state surface, labeled as nonlinear emission, followed by Raman-like dipole couplings. Two fourwave mixing energy level (FWMEL) diagrams together with the rules of Lee and Albrecht ${ }^{24}$ which were meant for continuous wave interactions gave an expression for RINE that yielded dispersive lines. By removing the term that gave rise to dispersion, reconstructed spectra with Lorentzian line shapes were obtained which decayed with a 260 fs time constant. The decay was attributed to the dynamical motion of the excited state wave packet along the many low-frequency modes from the Franck-Condon region $(\mathrm{H})$ to the photochemically active $\mathrm{I}_{460}$ region.

Here, we present the theory of FSRS of BR* in Sec. II. We argue that the actinic pump energy, although on resonance with $\mathrm{BR}^{*}$, is not too high in the experiment of $\mathrm{McCa}-$ mant et al. $^{11}$ and so each molecule would only have a few modes, typically the low frequency ones, excited while most modes are in the ground vibrational state. Over a large collection of molecules, the FSRS of $\mathrm{BR}^{*}$ which detects individual vibrational modes appears to be taking place effectively from the $v=0$ state of each observed mode on the $S_{1}$ surface. We use FWMEL diagrams, and there are eight of them which can be grouped as SRS(I), SRS(II), IRS(I), and IRS(II), to describe FSRS from BR*. It is then possible to identify the diagrams pertaining to RINE and others that are related to it. In Sec. III, we show how the experimental results are analyzed for the FSRS contribution from BR* only, and present calculations using the 29-mode model of Myers et $a .^{25}$ for the ground and excited state surfaces of BR. We are able to show that the dispersive line shapes arise from the IRS(I) term, and not from RINE. We conclude in Sec. IV.

\section{THEORY}

Myers et al. $^{25}$ have calculated the 29-mode harmonic ground and excited state surface parameters (Table I) for BR in the Franck-Condon region, from observed Raman spectra, with zero-zero energy of $17000 \mathrm{~cm}^{-1}$. For each mode, it is assumed that the frequency in the ground and excited electronic state is the same. This should be quite a good assumption since we are dealing with vibrations in the range of 800-1700 $\mathrm{cm}^{-1}$ in the FSRS experiment, and these correspond to hydrogen out-of-plane (HOOP) wags, C-C stretches, $\mathrm{CCH}$ in-plane rocks, and combination $\mathrm{C}=\mathrm{C}$ stretches where sigma bonds predominate, in a $\pi \rightarrow \pi^{*}$ transition with photoisomerization along a low frequency reaction coordinate of about $50 \mathrm{~cm}^{-1} .^{26,27}$

The $500 \mathrm{~nm}, 35 \mathrm{fs}$, actinic pump (ac) used in the BR experiment delivers photons largely in the energy range $20000 \pm 500 \mathrm{~cm}^{-1}$. Since the zero-zero energy between $S_{1}$ and $S_{0}$ is $17000 \mathrm{~cm}^{-1}$, it means that vibrational states between 2500 and $3500 \mathrm{~cm}^{-1}$ above the zero point energy of $\mathrm{S}_{1}$ will have amplitude with the actinic pump. Using the 29 modes in Table I, it can be shown that there are 105095 vibrational states within that energy range. When we analyze the quantum numbers of all these states, we find that $>95 \%$ of the states are in the $\mathrm{v}=0$ state for modes of frequencies $>1200 \mathrm{~cm}^{-1},>90 \%$ are in $\mathrm{v}=0$ for modes of frequencies $900-1200 \mathrm{~cm}^{-1}$, and $>85 \%$ are in $\mathrm{v}=0$ for modes of frequencies 800-900 $\mathrm{cm}^{-1}$. Since the FSRS spectra are taken in the frequency range of $800-1800 \mathrm{~cm}^{-1}$, the net result is that we are observing FSRS largely from $v=0$ for each of the 
TABLE I. Excited state parameters for $\mathrm{BR}_{568}$

\begin{tabular}{|c|c|c|}
\hline Vibrations & $\begin{array}{l}\text { Frequency } \omega_{r} \\
\quad\left(\mathrm{~cm}^{-1}\right)\end{array}$ & $\begin{array}{l}\text { Displacement } \\
\qquad\left|\Delta_{r}\right|\end{array}$ \\
\hline \multirow[t]{8}{*}{$\mathrm{CCC}$ torsions and bends } & 187 & 0.85 \\
\hline & 266 & 0.46 \\
\hline & 284 & 0.23 \\
\hline & 402 & 0.29 \\
\hline & 452 & 0.18 \\
\hline & 497 & 0.14 \\
\hline & 529 & 0.12 \\
\hline & 557 & 0.18 \\
\hline \multirow[t]{5}{*}{ HOOP wags } & 830 & 0.16 \\
\hline & 843 & 0.13 \\
\hline & 882 & 0.19 \\
\hline & 959 & 0.15 \\
\hline & 1008 & 0.42 \\
\hline \multirow[t]{4}{*}{$\mathrm{C}-\mathrm{C}$ stretches } & 1170 & 0.42 \\
\hline & 1201 & 0.38 \\
\hline & 1215 & 0.28 \\
\hline & 1253 & 0.17 \\
\hline \multirow[t]{8}{*}{$\mathrm{CCH}$ in plane rocks } & 1273 & 0.20 \\
\hline & 1304 & 0.14 \\
\hline & 1321 & 0.17 \\
\hline & 1333 & 0.08 \\
\hline & 1349 & 0.20 \\
\hline & 1379 & 0.09 \\
\hline & 1448 & 0.13 \\
\hline & 1457 & 0.13 \\
\hline \multirow[t]{4}{*}{$\mathrm{C}=\mathrm{C}$ stretches } & 1527 & 0.70 \\
\hline & 1580 & 0.15 \\
\hline & 1600 & 0.13 \\
\hline & 1640 & 0.21 \\
\hline
\end{tabular}

modes. We do not need to invoke intramolecular vibrational relaxation down to the ground vibrational state $(0,0, \ldots, 0)$ on $\mathrm{BR}^{*}$ at all which nevertheless does take place, and in $\mathrm{BR}^{*}$ it is thought to be ultrafast as observed in the $<30$ fs growth in the redshifted stimulated emission band. ${ }^{3}$ The FSRS that follows the actinic pump uses a $809 \mathrm{~nm}, 3.5 \mathrm{ps}$, Raman pump (pu) and a (840-960 nm) ultrashort probe pulse (pr) with energies that fall within a strong stimulated emission band from BR* $\left(S_{1}\right)$ to BR $\left(S_{0}\right),{ }^{3}$ and so we can use just these two surfaces in our FSRS calculations. The Raman pump and probe pulses couple $S_{1}$ to short-lived vibrational states on $S_{0}$ which we will explain below. Only the Stokes spectrum relative to the Raman pump frequency is measured in the probe pulse direction.

The theory of FSRS from the ground state surface has been given earlier in terms of eight Feynman dual time-line diagrams. ${ }^{19,22}$ Here, we use the familiar FWMEL diagrams of Lee and Albrecht ${ }^{24}$ to describe FSRS from the excited state surface applicable to $\mathrm{BR}^{*}$. The eight diagrams contributing to FSRS, ${ }^{19,22}$ grouped as SRS, SRS(I) and SRS(II), and IRS, IRS(I) and IRS(II), are shown in Fig. 2. For each diagram, time progresses from left to right; a solid arrow denotes interaction on the ket, a dash arrow denotes interaction on the bra, and the wavy line at time $t$ denotes closure between bra and ket to give the third-order polarization. The gray bar indicates the broadband probe or broadband polar-

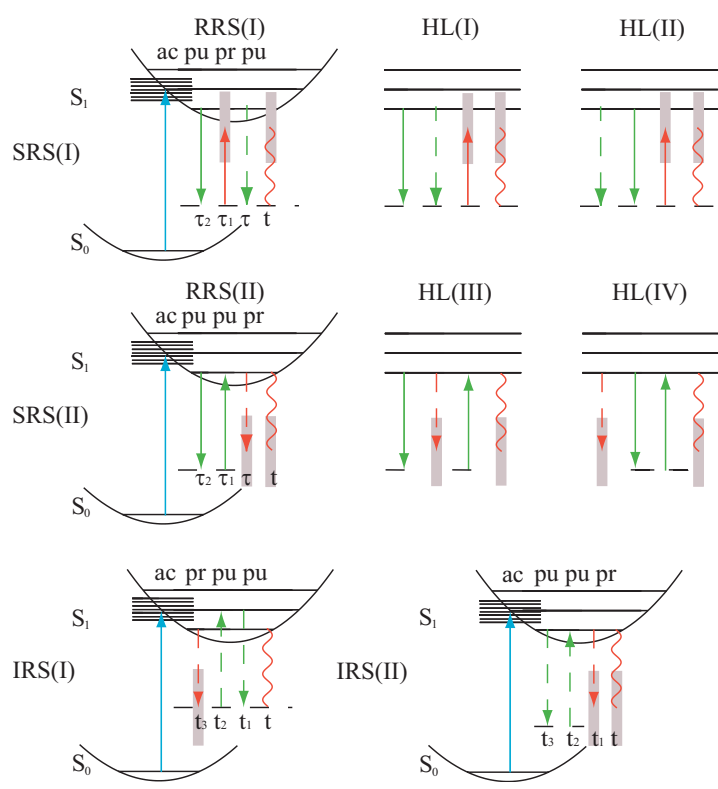

FIG. 2. FWMEL diagrams for the eight terms in FSRS relevant to the BR experiment, after an actinic (ac) pump prepares a wave packet on $S_{1}$. Time increases from left to right. Solid arrows correspond to ket interaction and dashed arrows for the bra. The Raman pump (pu) is to the blue of the probe (pr) pulse. SRS(I) comprises three diagrams: RRS(I), HL(I), and HL(II), with the bra interaction by the Raman pump at times $\tau, \tau_{1}$, and $\tau_{2}$, respectively. For HL(I) and HL(II), there can be intermediate population on $\mathrm{S}_{0}$ between $\left(\tau_{1}, \tau\right)$. Similarly, SRS(II) has three diagrams where the bra interaction is by the probe pulse. IRS(I) and IRS(II) have one diagram each, where all the interactions with the Raman pump and probe pulses are on the bra.

ization, if it is on the wavy line. Looking at the end of wavy line representing closure for the third-order polarization, it can be seen that the three diagrams for SRS(I)_RRS(I), HL(I), and HL(II) - are not resonant with any final vibrational state on $\mathrm{S}_{1}$ on the Stokes side of the Raman pump and so we would expect the SRS(I) spectrum to be broad and lacking in any vibrational features. In the three diagrams for SRS(II)_RRS(II), HL(III), and HL(IV)— the Raman pump takes the molecule to assumed short-lived vibrational states relatively high in energy on $\mathrm{S}_{0}$, and the end of the probe arrow will also engage similar short-lived vibrational states on $\mathrm{S}_{0}$ on the Stokes side, and the wavy line closure will be broadband. The same applies for IRS(II). These relatively high energy states of $S_{0}$ have many populated low frequency modes not in the observed range of $800-1800 \mathrm{~cm}^{-1}$ that can take the amplitude of the wave packet coming from $\mathrm{S}_{1}$ away from the Franck-Condon region quickly, and the correlation function for these low frequency modes is accounted for by a relatively small $\gamma_{0}^{-1}$ with fast decay, which indirectly makes the vibrational states for the observed modes appear short lived. $^{28,29}$ Thus SRS(II) and IRS(II) would give smooth spectra lacking in vibrational features. On the other hand, the diagram for IRS(I) shows resonance for the third-order polarization and coherence between $v=0$ and $v=1$ states on $S_{1}$ for each mode, and so we can expect to see vibrational lines for the modes of $S_{1}$ in the IRS(I) spectrum.

The RINE mechanism is described by McCamant et al. with a one-dimensional model. ${ }^{11}$ It assumes that the actinic pump prepares a stationary state $|n\rangle$ on $\mathrm{S}_{1}$, and a cw Raman pump matches the energy gap between $|n\rangle$ on $S_{1}$ and another 
vibrational state $\left|n^{\prime}\right\rangle$ on $\mathrm{S}_{0}$, followed by a cw probe that takes $\langle n|$ on $\mathrm{S}_{1}$ to $\left\langle n^{\prime}+1\right|$ on $\mathrm{S}_{0}$ thus creating a vibrational coherence $\left|n^{\prime}\right\rangle\left\langle n^{\prime}+1\right|$ on $\mathrm{S}_{0}$. These assumptions are not really consistent with the FSRS experiment that uses a picosecond Raman pump and a femtosecond probe, but nevertheless RINE as described can be represented by our HL(III) and HL(IV) diagrams where instead of short-lived states for the higher energy levels on $S_{0}$ we have long-lived real states, with a $\left|n^{\prime}\right\rangle\left\langle n^{\prime}+1\right|$ vibrational coherence created on $\mathrm{S}_{0}$ between times $\left(\tau_{2}, \tau_{1}\right)$ by the Raman pump acting on the ket and the probe field acting on the bra for HL(III), and a reversal of time ordering for HL(IV), in an emissionlike process. This is the "nonlinear emission," and it is followed by Raman-like dipole couplings where the Raman pump takes the ket $\left|n^{\prime}\right\rangle$ on $S_{0}$ to $S_{1}$ and a dipole coupling closure between the ket now on $\mathrm{S}_{1}$ and the bra $\left\langle n^{\prime}+1\right|$ on $\mathrm{S}_{0}$ with resultant polarization in the probe direction. Of significance are the dipole couplings between a vibrational state $|n\rangle$ on $\mathrm{S}_{1}$ and a pair of vibrational states $\left|n^{\prime}\right\rangle$ and $\left|n^{\prime}+1\right\rangle$ on $\mathrm{S}_{0}$ by the Raman pump and probe pulses, respectively, which are seen in the HL(III)and HL(IV) diagrams, but they are also present in the RRS(II) and IRS(II) diagrams. The RRS(II), HL(III), and HL(IV) diagrams are related and differ in only whether the probe field interaction on the bra comes after, in between, or before the two Raman pump field interactions on the ket, respectively, and hence they are grouped under the SRS(II) process. The IRS(II) diagram has been described as nonlinear emission elsewhere. ${ }^{27}$ All these diagrams can have the same spectral pattern.

The Raman gain cross section for FSRS is given $b^{22}$

$$
\sigma_{\mathrm{RG}}(\omega)=-\frac{4 \pi}{3 \epsilon_{0} c n} \omega \operatorname{Im}\left\{P_{\mathrm{diff}}^{(3)}(\omega) / E_{\mathrm{pr}}(\omega)\right\},
$$

where $\epsilon_{0}$ is the vacuum permittivity, $c$ is the speed of light, $n$ is the refractive index, $E_{\mathrm{pr}}(\omega)$ is the spectrum of the probe pulse which is given by

$$
E_{\mathrm{pr}}(\omega)=\frac{1}{2 \pi} \int_{-\infty}^{\infty} e^{i \omega t} E_{\mathrm{pr}}(t) d t,
$$

$P_{\text {diff }}^{(3)}(\omega)$ is the Fourier transform of the difference polarization with and without the Raman pump that radiates in the probe pulse direction,

$$
P_{\mathrm{diff}}^{(3)}(\omega)=\frac{1}{2 \pi} \int_{-\infty}^{\infty} e^{i \omega t} P_{\mathrm{diff}}^{(3)}(t) d t
$$

and the difference third-order polarization is given by

$$
\begin{aligned}
P_{\text {diff }}^{(3)}(t) & =P_{\text {pump on }}^{(3)}(t)-P_{\text {pump off }}^{(3)}(t) \\
& =P_{\mathrm{SRS}(\mathrm{I})}^{(3)}(t)+P_{\mathrm{SRS}(\mathrm{II})}^{(3)}(t)+P_{\mathrm{IRS}(\mathrm{I})}^{(3)}(t)+P_{\mathrm{IRS}(\mathrm{II})}^{(3)}(t) .
\end{aligned}
$$

We have an $N$-dimensional separable system. The Hamiltonians for $S_{1}$ and $S_{0}$ are sums of one-dimensional Hamiltonians,

$$
\begin{aligned}
& \mathbf{h}_{1}=\sum_{r=1}^{N} h_{1 r}+E_{10}, \\
& \mathbf{h}_{0}=\sum_{r=1}^{N} h_{0 r},
\end{aligned}
$$

where $h_{1 r}$ and $h_{0 r}$ are one-dimensional Hamiltonians in mode $Q_{r}$ on $\mathrm{S}_{1}$ and $\mathrm{S}_{0}$, and $E_{10}$ is the energy gap between the minima of the two multidimensional potential energy surfaces. The initial wave packet is a product of onedimensional wave function in each mode $r$ on the $S_{1}$ surface, which in the case of $\mathrm{BR}^{*}$ are all ground vibrational wave functions,

$$
\left|\psi_{1}(\mathbf{Q}, 0)\right\rangle=\prod_{r=1}^{N}\left|\psi_{1 r 0}\left(Q_{r}, 0\right)\right\rangle .
$$

We assume that the phenomenological damping matrix can be written as $\gamma_{i j}=(1 / 2)\left(\gamma_{i}+\gamma_{j}\right)$, where $\gamma_{i}$ is the inverse lifetime on electronic state $i$, and constant transition dipole moment $\mu_{10}$ (Condon approximation). ${ }^{21,27}$ The respective third-order polarizations can then be read off the diagrams in the usual manner, ${ }^{22}$

$$
\begin{aligned}
P_{\mathrm{SRS}(\mathrm{I})}^{(3)}(t)= & \left(\frac{i}{\hbar}\right)^{3} \int_{0}^{t} d \tau_{1} \int_{0}^{\tau_{1}} d \tau_{2} \int_{0}^{t} d \tau e^{-\gamma_{1} \tau / 2 \hbar-\gamma_{0}(t-\tau) / 2 \hbar-\gamma_{1}\left(t-\tau_{1}\right) / 2 \hbar-\gamma_{0}\left(\tau_{1}-\tau_{2}\right) / 2 \hbar-\gamma_{1} \tau_{2} / 2 \hbar} e^{i E_{10} \tau / \hbar} \\
& \times E_{\mathrm{pu}}\left(\tau ; T_{\mathrm{pu}}\right) e^{-i E_{10}\left(t-\tau_{1}\right) / \hbar} E_{\mathrm{pr}}\left(\tau_{1} ; T_{\mathrm{pr}}\right) E_{\mathrm{pu}}^{*}\left(\tau_{2} ; T_{\mathrm{pu}}\right) e^{-i E_{10} \tau_{2} / \hbar}\left|\mu_{10}\right|^{4} \prod_{r} S_{r}\left(t, \tau_{1}, \tau_{2}, \tau\right), \\
P_{\mathrm{SRS}(\mathrm{II})}^{(3)}(t)= & \left(\frac{i}{\hbar}\right)^{3} \int_{0}^{t} d \tau_{1} \int_{0}^{\tau_{1}} d \tau_{2} \int_{0}^{t} d \tau e^{-\gamma_{1} \tau / 2 \hbar-\gamma_{0}(t-\tau) / 2 \hbar-\gamma_{1}\left(t-\tau_{1}\right) / 2 \hbar-\gamma_{0}\left(\tau_{1}-\tau_{2}\right) / 2 \hbar-\gamma_{1} \tau_{2} / 2 \hbar} e^{i E_{10} \tau / \hbar} \\
& \times E_{\mathrm{pr}}\left(\tau ; T_{\mathrm{pr}}\right) e^{-i E_{10}\left(t-\tau_{1}\right) / \hbar} E_{\mathrm{pu}}\left(\tau_{1} ; T_{\mathrm{pu}}\right) E_{\mathrm{pu}}^{*}\left(\tau_{2} ; T_{\mathrm{pu}}\right) e^{-i E_{10} \tau_{2} / \hbar}\left|\mu_{10}\right|^{4} \prod_{r} S_{r}\left(t, \tau_{1}, \tau_{2}, \tau\right),
\end{aligned}
$$




$$
\begin{aligned}
P_{\mathrm{IRS}(\mathrm{I})}^{(3)}(t)= & \left(\frac{i}{\hbar}\right)^{3} \int_{0}^{t} d t_{1} \int_{0}^{t_{1}} d t_{2} \int_{0}^{t_{2}} d t_{3} e^{-\gamma_{1} t_{3} / 2 \hbar-\gamma_{0}\left(t_{2}-t_{3}\right) / 2 \hbar-\gamma_{1}\left(t_{1}-t_{2}\right) / 2 \hbar-\left(t_{1}-t_{2}\right) / T_{2}-\gamma_{0}\left(t-t_{1}\right) / 2 \hbar-\gamma_{1} t / 2 \hbar} e^{i E_{10} t_{3} / \hbar} \\
& \times E_{\mathrm{pr}}\left(t_{3} ; T_{\mathrm{pr}}\right) E_{\mathrm{pu}}^{*}\left(t_{2} ; T_{\mathrm{pu}}\right) e^{i E_{10}\left(t_{1}-t_{2}\right) / \hbar} E_{\mathrm{pu}}\left(t_{1} ; T_{\mathrm{pu}}\right) e^{-i E_{10} t / \hbar}\left|\mu_{10}\right|^{4} \prod_{r} I_{r}\left(t, t_{1}, t_{2}, t_{3}\right),
\end{aligned}
$$

and

$$
\begin{aligned}
P_{\mathrm{IRS}(\mathrm{II})}^{(3)}(t)= & \left(\frac{i}{\hbar}\right)^{3} \int_{0}^{t} d t_{1} \int_{0}^{t_{1}} d t_{2} \int_{0}^{t_{2}} d t_{3} e^{-\gamma_{1} t_{3} / 2 \hbar-\gamma_{0}\left(t_{2}-t_{3}\right) / 2 \hbar-\gamma_{1}\left(t_{1}-t_{2}\right) / 2 \hbar-\gamma_{0}\left(t-t_{1}\right) / 2 \hbar-\gamma_{1} t / 2 \hbar} e^{i E_{10} t_{3} / \hbar} E_{\mathrm{pu}}\left(t_{3} ; T_{\mathrm{pu}}\right) \\
& \times E_{\mathrm{pu}}^{*}\left(t_{2} ; T_{\mathrm{pu}}\right) e^{i E_{10}\left(t_{1}-t_{2}\right) / \hbar} E_{\mathrm{pr}}\left(t_{1} ; T_{\mathrm{pr}}\right) e^{-i E_{10} / \hbar}\left|\mu_{10}\right|^{4} \prod_{r} I_{r}\left(t, t_{1}, t_{2}, t_{3}\right) .
\end{aligned}
$$

Only the IRS(I) diagram shows vibrational coherence on $\mathrm{S}_{1}$; so, in Eq. (10), in addition to the electronic lifetimes $\gamma_{i}$, we added a vibrational coherence $|0\rangle\langle 1|$ dephasing time $T_{2}$ in $\mathrm{BR}^{*}$ for evolution between $\left(t_{2}, t_{1}\right)$, assuming that it is the same for all modes. The Raman pump (pu) or probe (pr) pulse (electric field) is commonly taken to be of the form

$$
E_{\mathrm{pu} / \mathrm{pr}}\left(t ; T_{\mathrm{pu} / \mathrm{pr}}\right)=E_{0, \mathrm{pu} / \mathrm{pr}} g_{\mathrm{pu} / \mathrm{pr}}\left(t ; T_{\mathrm{pu} / \mathrm{pr}}\right) e^{-i \omega_{\mathrm{pu} / \mathrm{pr}} t},
$$

where $E_{0, \mathrm{pu} / \mathrm{pr}}$ is a constant, and the Gaussian pulse envelope $g_{\mathrm{pu} / \mathrm{pr}}\left(t ; T_{\mathrm{pu} / \mathrm{pr}}\right)$ is given by

$$
g_{\mathrm{pu} / \mathrm{pr}}\left(t ; T_{\mathrm{pu} / \mathrm{pr}}\right)=\exp \left(-2 \ln 2 \frac{\left(t-T_{\mathrm{pu} / \mathrm{pr}}\right)^{2}}{\Delta_{\mathrm{pu} / \mathrm{pr}}^{2}}\right),
$$

giving a laser pulse centered at $T_{\mathrm{pu} / \mathrm{pr}}$, with intensity proportional to $\left|g_{\mathrm{pu} / \mathrm{pr}}\left(t ; T_{\mathrm{pu} / \mathrm{pr}}\right)\right|^{2}$ with full-width at half maximum of $\Delta_{\mathrm{pu} / \mathrm{pr}}$. For each mode $r$, the four-time correlation functions are defined as

$$
\begin{aligned}
& S_{r}\left(t, \tau_{1}, \tau_{2}, \tau\right) \\
&=\left\langle\psi_{1 r 0}\left(Q_{r}, 0\right)\right| e^{i h_{1 r} \tau / \hbar} e^{i h_{0 r}(t-\tau) / \hbar} \\
& \times e^{-i h_{1 r}\left(t-\tau_{1}\right) / \hbar} e^{-i h_{0 r}\left(\tau_{1}-\tau_{2}\right) / \hbar} e^{-i h_{1 r} \tau_{2} / \hbar}\left|\psi_{1 r 0}\left(Q_{r}, 0\right)\right\rangle,
\end{aligned}
$$

and

$$
\begin{aligned}
& I_{r}\left(t, t_{1}, t_{2}, t_{3}\right) \\
&=\left\langle\psi_{1 r 0}\left(Q_{r}, 0\right)\right| e^{i h_{1 r} t_{3} / \hbar} e^{i h_{0 r}\left(t_{2}-t_{3}\right) / \hbar} \\
& \times e^{i h_{1 r}\left(t_{1}-t_{2}\right) / \hbar} e^{i h_{0 r}\left(t-t_{1}\right) / \hbar} e^{-i h_{1 r} t / \hbar}\left|\psi_{1 r 0}\left(Q_{r}, 0\right)\right\rangle .
\end{aligned}
$$

The correlation functions can be computed analytically for a pair of displaced harmonic oscillators without frequency changes between the excited and ground state potentials. ${ }^{22}$

\section{EXPERIMENTAL RESULTS, CALCULATIONS, AND DISCUSSION}

\section{A. Experimental results}

The experimental results have been obtained by $\mathrm{McCa}$ mant et al. $^{11}$ where an actinic pump pulse $(500 \mathrm{~nm}, 35 \mathrm{fs})$ first prepares a mixture of $\mathrm{BR}(\sim 91 \%)$ and $\mathrm{BR}^{*}(\sim 9 \%)$ followed by FSRS with a (809 nm, $3.5 \mathrm{ps)} \mathrm{Raman} \mathrm{pump} \mathrm{and} \mathrm{a}$ (840-960 nm) ultrashort probe pulse. The Raman gain spec- trum for the mixture of $\mathrm{BR}$ and $\mathrm{BR}^{*}$ is given by the ratio of the Raman pump-on probe spectrum over the Raman pumpoff probe spectrum. The Raman gain spectra for various delay times between the actinic pump and probe pulses are shown in Fig. 3, together with the Raman gain spectrum of the BR ground state. We then fitted a ninth-order polynomial baseline to each of the spectra and subtracted it from its baseline. For delay times of $40 \mathrm{fs}$ and greater, we also subtracted 0.91 times the ground state spectrum following the prescription of McCamant et al., ${ }^{11}$ while for the delay time of 0 fs we subtracted 0.955 times the ground state spectrum because the $\mathrm{BR}^{*}$ population would only be half of the peak value. The net result is the Raman gain spectra for BR* only, without the baselines, and these are shown in Fig. 4. Dispersive lines for about eight to nine vibrational modes can be seen clearly, with intensities more or less proportional to $\omega_{r}^{3} \Delta_{r}^{2}$, just as for the usual Raman intensities. The linewidths stay nearly the same for all time delays, and the intensity of the lines was deduced to decay with a time constant of $\sim 260$ fs based on the RINE model. ${ }^{11}$

\section{B. Calculations and discussion}

We first calculated the Raman gain spectrum for the ground state BR using the potential parameters in Table I with zero-zero energy $17000 \mathrm{~cm}^{-1}$, (809 nm, 3.5 ps) Raman pump, and a (900 nm, $10 \mathrm{fs)} \mathrm{probe} \mathrm{pulse.} \mathrm{It} \mathrm{is} \mathrm{off-resonance}$ FSRS, and the principal contribution is from the RRS(I) term. ${ }^{19,22}$ In principle, we do not need to use all 29 modes in Table I, but only those in the range of $800-1700 \mathrm{~cm}^{-1}$ relevant to the experiment; nevertheless, we included them all in the calculations and the results are the same. The shape of the ultrashort probe pulse is not critical so long as its spectrum spans the range 840-960 $\mathrm{nm}$ for observing the FSRS spectrum. As in the experiment, the probe precedes the Raman pump by $1 \mathrm{ps}$. The method for calculating the FSRS for polyatomics with harmonic potentials from the ground vibrational state is described in Niu et al. ${ }^{22}$ In this case, its offresonance FSRS where the contribution is wholly from the RRS(I) term with vibrational coherence $|0\rangle\langle 1|$ on $S_{0}$ and associated vibrational dephasing time $T_{2}$ that affects the linewidths. Since its FSRS from the stationary ground vibrational state, we set $\gamma_{0}=0$, and for off-resonance FSRS the 


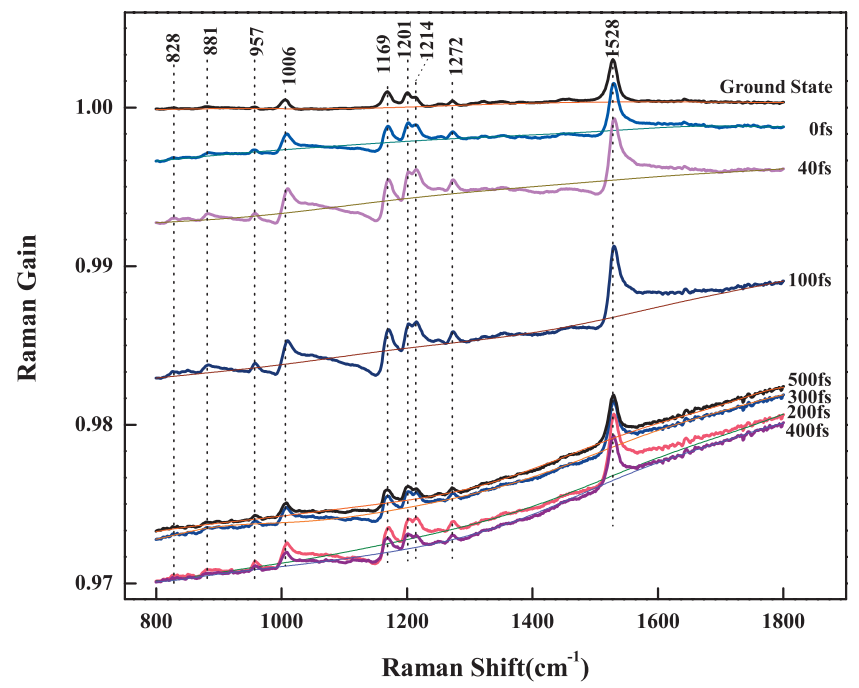

FIG. 3. FSRS Raman gain spectra of a mixture of $B R$ and $B R^{*}$ for various time delays between actinic pump and probe pulses with ninth-order polynomial baselines drawn in. The FSRS Raman gain spectrum of the ground state BR is also shown.

wave packet dynamics on the excited state $S_{1}$ is short lived by the uncertainty principle and so $\gamma_{1}$ is less important and we can set $\gamma_{1}=0 .{ }^{29,30}$ We chose $T_{2}$ on $S_{0}$ to be 600 fs to give linewidths comparable to the experimental results, ${ }^{11}$ as shown in Fig. 5. A longer dephasing time $T_{2}$, which is homogeneous damping, together with inhomogeneous damping due to fluctuations in the zero-zero energy $E_{10}$ can also give similar broadening in the spectral lines, and absolute resonance Raman intensities would be needed to distinguish between homogeneous and inhomogeneous broadening. ${ }^{25}$ There is generally excellent agreement between the calculated and experimental results which shows that the sepa-

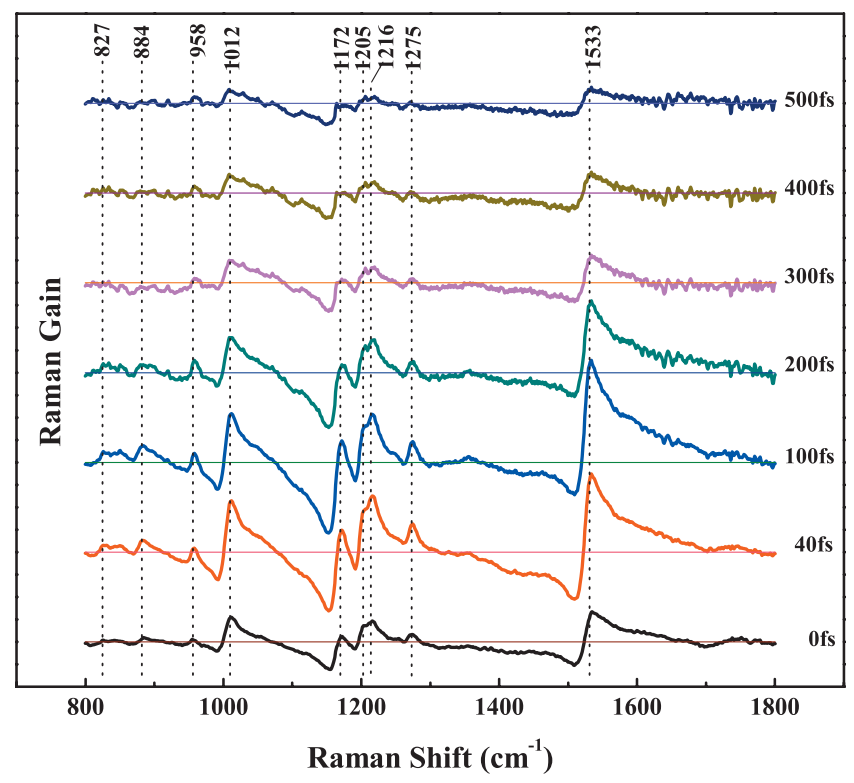

FIG. 4. FSRS Raman gain spectra for $\mathrm{BR}^{*}$ only (vertically offset) without the baselines at various time delays between actinic pump and probe pulses. These are obtained by subtracting the baselines followed by subtracting 0.91 times the ground state spectrum for delay times of $40 \mathrm{fs}$ and greater, while for the delay time of 0 fs we subtracted 0.955 times the ground state spectrum because the $\mathrm{BR}^{*}$ population would only be half of the peak value.

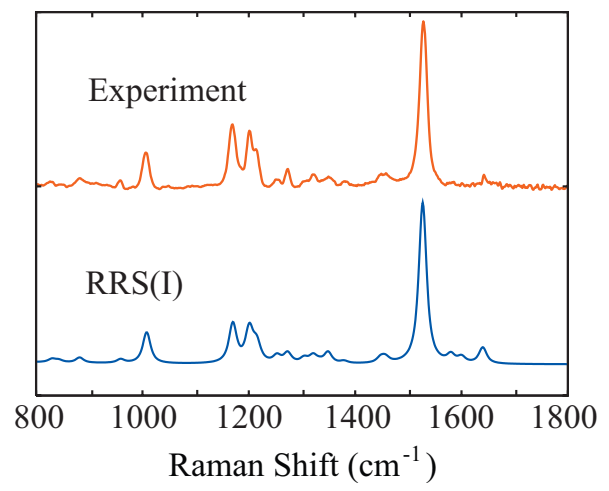

FIG. 5. Experimental and calculated results with the 29-mode model for the FSRS Raman gain of ground state BR. The calculated results are with the RRS(I) term as this is off-resonance FSRS and we used a vibrational coherence dephasing time $T_{2}$ on $\mathrm{S}_{0}$ of $600 \mathrm{fs}$.

rable harmonic model is applicable and the harmonic potential parameters are good. However, some lower intensity lines like the $1272 \mathrm{~cm}^{-1}$ line appear weaker and the $1640 \mathrm{~cm}^{-1}$ line appear stronger in the calculated results compared to the experiment. This may mean that the excited state displacements in Table I or the transition dipole moments for these modes need to be adjusted. The strengths of these lines, although of lower intensity, also show in the calculated FSRS Raman gain for BR* below.

The $500 \mathrm{~nm}, 35 \mathrm{fs}$, actinic pump prepares population on $\mathrm{BR}^{*}$ followed by FSRS with a pair of Raman pump and probe pulses as described above. The population prepared on $\mathrm{BR}^{*}$ would reach a peak by about $40 \mathrm{fs}$, and at 0 fs time delay between the actinic pump and probe, only half of the peak population is prepared. This accounts for why the Raman gain in Fig. 4 for 0 fs delay is only about half that for 40 fs delay. As explained in the theory above, the FSRS of BR* largely takes place from the ground vibrational state of each mode and so we could calculate it effectively from the multidimensional ground vibrational state of $S_{1}$. The calculations on FSRS from BR* were made with Eqs. (1)-(15) above. We have chosen $\gamma_{0}^{-1}=100$ fs to reflect the short lifetime in this relatively high-lying energy region of $S_{0}$ reached by the Raman pump/probe from $S_{1}$, where the available phase space is larger and the potential is anharmonic. The decay factor can be attributed to the many low frequency modes outside the observed range of $800-1800 \mathrm{~cm}^{-1}$ that can take the amplitude of the wave packet coming from $\mathrm{S}_{1}$ away from the Franck-Condon region quickly, and the correlation function from these low frequency modes has a fast decay. A longer lifetime in this high-lying energy region of $\mathrm{S}_{0}$ would lead to resolved vibrational states and noisy lines in the FSRS spectra from SRS(II) and IRS(II) processes as shown below, unlike the relatively smooth spectra observed in the experiment. The lifetime $\gamma_{1}^{-1}$ of $S_{1}$ was chosen to be $600 \mathrm{fs}$ and the vibrational dephasing time $T_{2}$ on $\mathrm{S}_{1}$ was taken to be infinite, to simulate the observed linewidths, but a longer lifetime $\gamma_{1}^{-1}$ and a shorter $T_{2}$ for $\mathrm{S}_{1}$ could give similar linewidths. It is reasonable for $\gamma_{1}^{-1}$, where we are starting with the lower vibrational states on $S_{1}$, to be larger than $\gamma_{0}^{-1}$, where we have dipole couplings with the more numerous and higher vibrational states of $\mathrm{S}_{0}$ in the FSRS of BR* The calculated results 

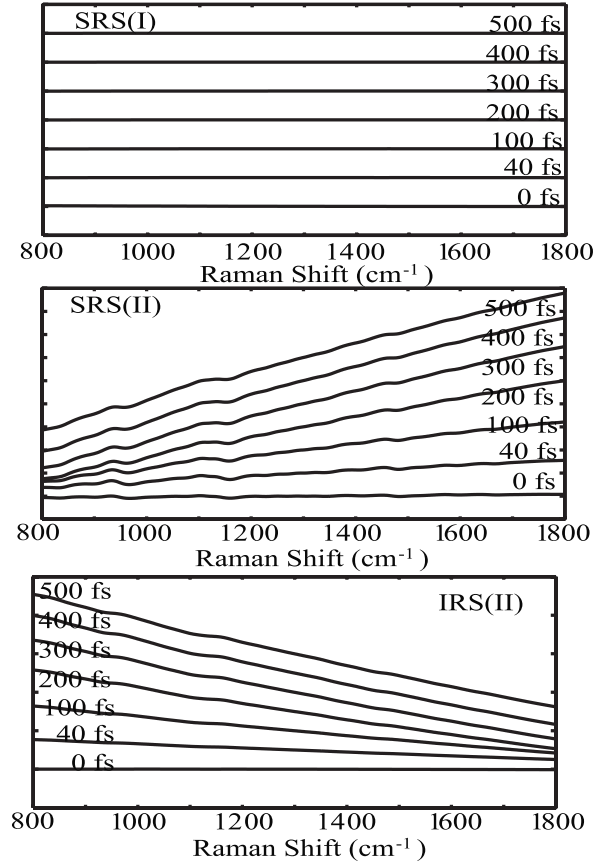

FIG. 6. Calculated results (vertically offset) for SRS(I), SRS(II), and IRS(II) in the Raman gain of $\mathrm{BR}^{*}$ for time delays between actinic pump and probe of $0-500 \mathrm{fs}$. Lifetimes used are $\gamma_{0}^{-1}=100 \mathrm{fs}, \gamma_{1}^{-1}=600 \mathrm{fs}$, and $T_{2}$ on $\mathrm{S}_{1}$ at infinity.

for SRS(I), SRS(II), and IRS(II) for time delays between actinic pump and probe of 0-500 fs are shown in Fig. 6, and they are all broad and without vibrational features which can be explained with the FWMEL diagrams in Fig. 2 where the wavy line closure for the polarization is broad. The SRS(I) lines are flat, and the SRS(II) and IRS(II) lines are decreasing in magnitude in the Stokes range of $800-1800 \mathrm{~cm}^{-1}$ due to the probe interaction getting further away from resonance with $\mathrm{S}_{0}$ as the Stokes frequency increases, as shown in Fig. 2 , and they tend to the zero baseline from the negative and positive side, respectively, with $180^{\circ}$ phase difference. All these spectra contribute only to the Raman gain baseline. However, the IRS(I) spectra, shown in Fig. 7, show a striking resemblance in the dispersive lines, in the linewidths, frequencies, and relative intensities, to the experimental results shown in Fig. 4. The IRS(I) process with its dispersive lines of the correct phase, with a negative dip on the low frequency side and rising sharply to a positive peak followed by a return to the baseline, from the vibrational coherence $|0\rangle\langle 1|$

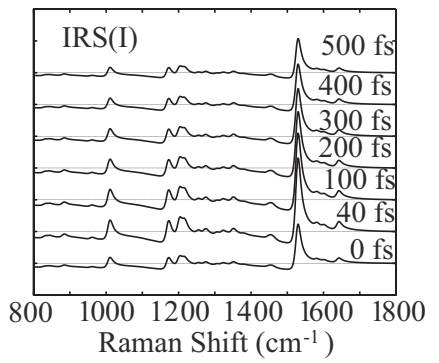

FIG. 7. Calculated results (vertically offset) for IRS(I), in the Raman gain of $\mathrm{BR}^{*}$ for time delays between actinic pump and probe of 0-500 fs. Lifetimes used are $\gamma_{0}^{-1}=100 \mathrm{fs}, \gamma_{1}^{-1}=600 \mathrm{fs}$, and $T_{2}$ on $\mathrm{S}_{1}$ at infinity. The dispersive line shapes resemble the experimental results shown in Fig. 4.
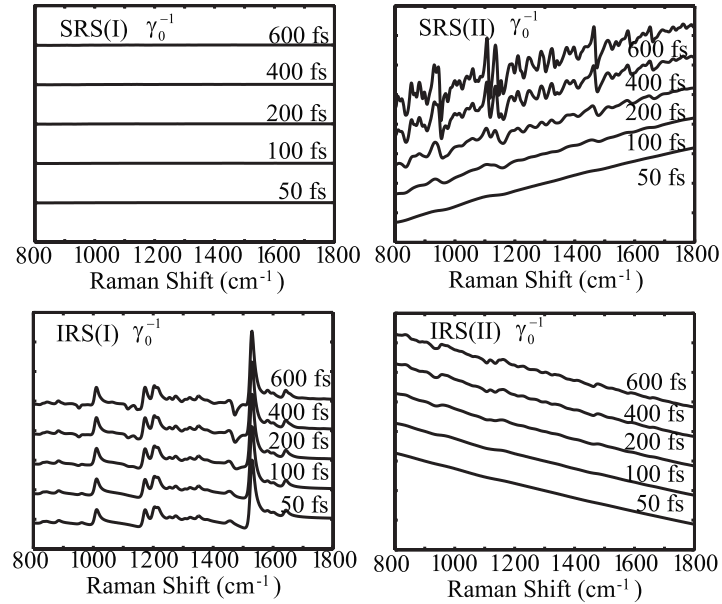

FIG. 8. Calculated results (vertically offset) for SRS(I), SRS(II), IRS(I), and IRS(II) in the Raman gain of $\mathrm{BR}^{*}$ at a time delay of 100 fs with $\gamma_{1}^{-1}$ $=600 \mathrm{fs}, T_{2}$ on $\mathrm{S}_{1}$ at infinity, and for $\gamma_{0}^{-1}$ ranging from $50-600 \mathrm{fs}$.

on $\mathrm{S}_{1}$ is dominant for all delay times. All the observed modes are well reproduced, and it shows that RINE, included in SRS(II), is not the explanation, but rather it is IRS given by IRS(I) that gives rise to the dispersive lines. Dispersive lineshapes have been observed before in resonant IRS of rhodamine 640 with cw lasers. ${ }^{31}$

The $\sim 600$ fs lifetime on $S_{1}$ here is comparable to the stimulated emission decay time, but it is longer than the rapid 260 fs decay estimated by McCamant et al. ${ }^{11}$ from reconstructed RINE peak areas. Our model here is different from the RINE model. A shorter lifetime $\gamma_{1}^{-1}$ on $S_{1}$ would lead to broader dispersive lines for each of the modes and nearby lines would coalesce. The effect of the lifetime $\gamma_{1}^{-1}$ of $\mathrm{S}_{1}$ and the dephasing time $T_{2}$ on the linewidths in the FSRS spectra will be discussed further below. Shorter lifetimes for $\gamma_{0}^{-1}$ gave identical results, and we will also discuss longer lifetimes below.

We would expect the RINE spectra, which are included in SRS(II), to show vibrational structures when the lifetime $\gamma_{0}^{-1}$ is increased, so that the short-lived states reached by the Raman pump/probe may turn into longer-lived states and it would be possible to create a vibrational coherence on $S_{0}$. In Fig. 8 we show the results for time delay of 100 fs and $\gamma_{0}^{-1}$ ranging from 50-600 fs, while keeping $\gamma_{1}^{-1}=600$ fs and $T_{2}$ at infinity. Indeed, vibrational structures begin to appear in the spectra for $\gamma_{0}^{-1} \geq 200$ fs in SRS(II) and IRS(II), and they are made up of fundamentals and combination bands espe-

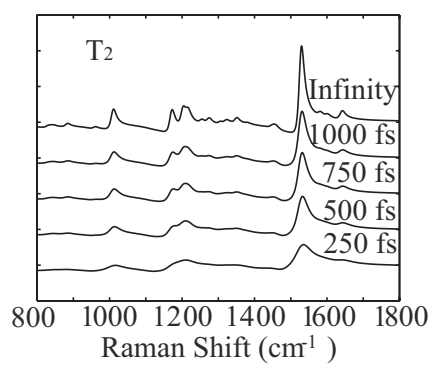

FIG. 9. Calculated results (vertically offset) for IRS(I) in the Raman gain of $\mathrm{BR}^{*}$ at a time delay of $100 \mathrm{fs}$ with $\gamma_{0}^{-1}=100 \mathrm{fs}, \gamma_{1}^{-1}=600 \mathrm{fs}$, and $T_{2}$ on $\mathrm{S}_{1}$ ranging from 250-infinity fs. 


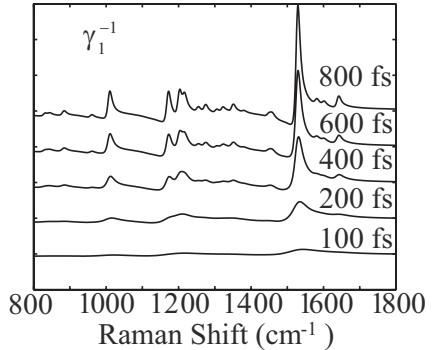

FIG. 10. Calculated results (vertically offset) for IRS(I) in the Raman gain of $\mathrm{BR}^{*}$ at a time delay of $100 \mathrm{fs}$ with $\gamma_{0}^{-1}=100 \mathrm{fs}, T_{2}$ on $\mathrm{S}_{1}$ at infinity, and $\gamma_{1}^{-1}$ ranging from $100-800 \mathrm{fs}$.

cially with the lower frequency modes. The dispersive lines in the IRS(I) spectra, however, remain at well defined frequencies of $\mathrm{BR}^{*}$ due to the ever present $|0\rangle\langle 1|$ vibrational coherence on $S_{1}$ for each mode. It can also be seen that the width of the dispersive lines in IRS(I) is rather independent of the lifetime $\gamma_{0}^{-1}$ on $\mathrm{S}_{0}$. The observed FSRS spectra would be the sum of SRS(I), SRS(II), IRS(I), and IRS(II), and since the baselines of the observed spectra are relatively smooth, we can rule out larger values for $\gamma_{0}^{-1} \geq 200$ fs because they can lead to a noisy SRS(II) component due to many possible vibrational resonances and a noisy baseline.

The width of the dispersive lines in IRS(I) depends on both the lifetime $\gamma_{1}^{-1}$ on $S_{1}$ as well as the $|0\rangle\langle 1|$ vibrational dephasing time $T_{2}$. In Fig. 9, we show the IRS(I) results for time delay of $100 \mathrm{fs}, \gamma_{1}^{-1}=600 \mathrm{fs}, \gamma_{0}^{-1}=100 \mathrm{fs}$, and $T_{2}$ ranging from 250-infinity fs. In Fig. 10, we show the IRS(I) results for time delay of $100 \mathrm{fs}, \gamma_{0}^{-1}=100 \mathrm{fs}, T_{2}$ at infinity, and $\gamma_{1}^{-1}$ ranging from $100-800 \mathrm{fs}$. Clearly, as $T_{2}$ or $\gamma_{1}^{-1}$ decreases, the dispersive linewidths get broader. A decay time constant of $\gamma_{1}^{-1}=600$ fs on $S_{1}$, or a longer $\gamma_{1}^{-1}$ plus a finite vibrational dephasing time $T_{2}$ of the order of a picosecond would also give dispersive linewidths that compare well with experiment.

\section{CONCLUSION}

FSRS of $\mathrm{BR}^{*}$ prepared by a $500 \mathrm{~nm}, 35$ fs actinic pump pulse effectively takes place from the $\mathrm{v}=0$ state of each of the modes in the observed Stokes range of $800-1800 \mathrm{~cm}^{-1}$ on $\mathrm{S}_{1}$. FWMEL diagrams are used to describe the FSRS process, and the eight diagrams can be grouped into SRS(I), SRS(II), IRS(I), and IRS(II). It is shown that RINE is equivalent to HL(III) + HL(IV) which is a subset of SRS(II). Calculations with the 29-mode model for BR show that the dispersive lines observed in the FSRS of $\mathrm{BR}^{*}$ come from IRS, IRS(I), with $|0\rangle\langle 1|$ vibrational coherence on the $S_{1}$ surface, and are not due to RINE with vibrational coherence on the $\mathrm{S}_{0}$ surface. In our model, the dipole couplings are from $\mathrm{S}_{1}$ to short-lived states, $\sim 100 \mathrm{fs}$, on the $\mathrm{S}_{0}$ surface, where the decay can be attributed to the correlation function from the many low frequency modes on $\mathrm{S}_{0}$ and $\mathrm{S}_{1}$ outside the observed range of $800-1800 \mathrm{~cm}^{-1}$ in the FSRS experiment. The decay time constant $\gamma_{1}^{-1}$ on $\mathrm{S}_{1}$ is estimated to be $\sim 600$ fs which is comparable to the stimulated emission decay time. A shorter $\gamma_{1}^{-1}$ of $260 \mathrm{fs}$ estimated by McCamant et $a l .{ }^{11}$ with the RINE model would lead to broader dispersive lines and nearby lines would coalesce contrary to the sharp dispersive lines observed in the experiment. We explained the origin of the dispersive lines. However, the time-resolved FSRS experiment with a $500 \mathrm{~nm}, 35 \mathrm{fs}$ actinic pump pulse with observation of the Stokes spectra in the $800-1800 \mathrm{~cm}^{-1}$ range is unable to provide direct observation of the dynamics of $\mathrm{BR}^{*}$ that may be happening along the low frequency modes and is not present in the current dispersive lines.

\section{ACKNOWLEDGMENTS}

We thank Professor David McCamant and Professor Richard Mathies for the experimental data on BR and many helpful discussions related to the manuscript. This work was supported by the Ministry of Education, Singapore, Research Grant No. T207B1222.

${ }^{1}$ R. A. Mathies, C. Brito Cruz, S. W. Pollard, and C. Shank, Science 240, 777 (1988).

${ }^{2}$ S. Shim, J. Dasgupta, and R. A. Mathies, J. Am. Chem. Soc. 131, 7592 (2009).

${ }^{3}$ Q. Zhong, S. Ruhman, M. Ottolenghi, N. Friedman, G. H. Atkinson, and J. K. Delaney, J. Am. Chem. Soc. 118, 12828 (1996).

${ }^{4}$ R. R. Birge, Annu. Rev. Phys. Chem. 41, 683 (1990).

${ }^{5}$ M. Ottolenghi and M. Sheves, Isr. J. Chem. 35, 193 (1995).

${ }^{6} \mathrm{H}$. Haupts, J. Tittor, and D. Oesterhelt, Annu. Rev. Biophys. Biomol. Struct. 367, 28 (1999).

${ }^{7}$ D. W. McCamant, P. Kukura, and R. A. Mathies, Appl. Spectrosc. 57, 1317 (2003).

${ }^{8}$ P. Kukura, D. W. McCamant, P. H. Davis, and R. A. Mathies, Chem. Phys. Lett. 382, 81 (2003).

${ }^{9}$ D. W. McCamant, P. Kukura, S. W. Yoon, and R. A. Mathies, Rev. Sci. Instrum. 75, 4971 (2004).

${ }^{10}$ P. Kukura, D. W. McCamant, and R. A. Mathies, J. Phys. Chem. A 108, 5921 (2004).

${ }^{11}$ D. W. McCamant, P. Kukura, and R. A. Mathies, J. Phys. Chem. B 109, 10449 (2005).

${ }^{12}$ P. Kukura, D. McCamant, S. Yoon, D. Wandschneider, and R. A. Mathies, Science 310, 1006 (2005).

${ }^{13}$ P. Kukura, S. Yoon, and R. A. Mathies, Anal. Chem. 78, 5952 (2006).

${ }^{14}$ P. Kukura, R. Frontiera, and R. A. Mathies, Phys. Rev. Lett. 96, 238303 (2006).

${ }^{15}$ P. Kukura, D. W. McCamant, and R. A. Mathies, Annu. Rev. Phys. Chem. 58, 461 (2007).

${ }^{16}$ M. Yoshizawa, Y. Hattori, and T. Kobayashi, Phys. Rev. B 49, 13259 (1994).

${ }^{17}$ M. Yoshizawa, H. Aoki, and H. Hashimoto, Phys. Rev. B 63, 180301(R) (2001).

${ }^{18}$ J. Dasgupta, R. R. Frontiera, K. C. Taylor, J. C. Lagarias, and R. A. Mathies, Proc. Natl. Acad. Sci. U.S.A. 106, 1784 (2009).

${ }^{19}$ Z. Sun, J. Lu, D. H. Zhang, and S. Y. Lee, J. Chem. Phys. 128, 144114 (2008).

${ }^{20}$ Z. Sun, X. Q. Qiu, J. Lu, D. H. Zhang, and S. Y. Lee, J. Raman Spectrosc. 39, 1568 (2008).

${ }^{21}$ Y. R. Shen, Phys. Rev. B 9, 622 (1974).

${ }^{22}$ K. Niu, S. Cong, and S. Y. Lee, J. Chem. Phys. 131, 054311 (2009).

${ }^{23}$ Z. Sun, B. Fu, D. H. Zhang, and S. Y. Lee, J. Chem. Phys. 130, 044312 (2009).

${ }^{24}$ D. Lee and A. C. Albrecht, in Advances in Infrared and Raman Spectroscopy, edited by J. H. Clark and R. E. Hester (Wiley Heyden, London, 1985), Vol. 12, pp. 179-213.

${ }^{25}$ A. B. Myers and R. A. Mathies, Biological Applications of Raman Spectroscopy: Resonance Raman Spectra of Polyenes and Aromatics (Wiley, New York, 1987), Vol. 2, pp. 1-58.

${ }^{26}$ W. T. Pollard, C. H. Brito Cruz, C. V. Shank, and R. A. Mathies, J. Chem. Phys. 90, 199 (1989). 
${ }^{27}$ W. T. Pollard, S. Y. Lee, and R. A. Mathies, J. Chem. Phys. 92, 4012 (1990).

${ }^{28}$ E. J. Heller, Acc. Chem. Res. 14, 368 (1981).

${ }^{29}$ E. J. Heller, R. L. Sundberg, and D. Tannor, J. Phys. Chem. 86, 1822
(1982).

${ }^{30}$ S. Y. Lee and E. J. Heller, J. Chem. Phys. 71, 4777 (1979).

${ }^{31}$ M. Takayanagi, H. Hamaguchi, and M. Tasumi, J. Chem. Phys. 89, 3945 (1988). 